\title{
Does lateralisation of the centre of rotation in reverse shoulder arthroplasty avoid scapular notching? Clinical and radiological review of one hundred and forty cases with forty five months of follow-up
}

\author{
Denis Katz $^{1} \cdot$ Philippe Valenti $^{1} \cdot$ Jean Kany $^{1} \cdot$ Kamil Elkholti $^{1} \cdot$ Jean-David Werthel $^{1}$
}

Received: 1 May 2015 / Accepted: 8 August 2015 /Published online: 4 September 2015

(C) The Author(s) 2015. This article is published with open access at Springerlink.com

\begin{abstract}
Purpose Our purpose was to evaluate the influence of metallic lateralisation of the centre of rotation (COR) in reverse shoulder arthroplasty (RSA) on the incidence of scapular notching and its eventual clinical and radiological consequences.

Methods We analysed 140 RSAs with a lateralised design (Arrow, FH Orthopedics, Mulhouse, France) implanted for massive rotator cuff tear with/without arthritis. Mean followup was 45 months (range 24-120, standard deviation 20). Patients were evaluated clinically using the Constant and Murley (Clin Orthop Relat Res 214:160-164, 1987) score and active range of motion (ROM) and radiologically using standard anteroposterior and axillary view. Scapular notching was assessed according to Sirveaux classification Simovitch et al. (J Bone Joint Surg Am, 89:588-600, 2007), and patients were separated into two groups (scapular notch/no scapular notch) and compared.

Results Forty-one notches (29\%) were found: 20 grade 1, 18 grade 2, and three grade 3 . The latter three patients had a follow-up of 44, 70 and 84 months, respectively, and the scapular notch did not evolve in the final two years. Better preoperative function was significantly associated with scapular notching ( $p<0,05$ for flexion and abduction), but no final clinical differences in ROM and Constant score were found between groups. A body mass index (BMI) $<30$ increased the risk of scapular notching, which was observed in $43 \%$ of patients with a $\mathrm{BMI}<30$ and $30 \%$ of patients with a $\mathrm{BMI}>$ $30 p=0,048)$. Pre-operative narrowing of the subacromial
\end{abstract}

Denis Katz

denis.katz@wanadoo.fr

1 Douar Gwen, 56270 Ploemeur, France space increased the risk of scapular notching, but age or gender showed no influence. However, there was a significantly greater number of scapular notches in patients operated on the dominant side $(p=0,04)$. No significant difference in lateral offset was found between groups $(p=0,99)$. Glenoid implantation in an excessively high position $(p=0,033)$ and absence of inferior tilt $(p=0,0029)$ were significantly associated with scapular notching.

Conclusions In this series, metallic lateralisation of the COR in RSA did not impair clinical results, with patients achieving good flexion recovery ROM increase in rotations. Metallic lateralisation of the COR in RSA leads to a lower incidence of scapular notching ( $29 \%$ ) compared with previously reported results using other arthroplastic systems with a more medialised COR. However, although scapular notching was not totally eliminated, those that were found did not evolve over time. Several factors increased the incidence of scapular notching: $\mathrm{BMI}<30$, better preoperative ROM, an excessively high glenoid implant and absence of inferior tilt.

Keywords Reverse shoulder arthroplasty · Scapular notching $\cdot$ Clinical outcome $\cdot$ Rotator cuff tear $\cdot$ Arthritis . Cuff-tear arthropathy

\section{Introduction}

In the last 30 years of orthopaedic shoulder surgery, one of the most impressive technical improvements is the ability to restore good function to patients with massive irreparable cuff tears associated or not with arthritis. [20, 21] Grammont introduced the original concept of implanting the sphere on the glenoid side [19]. This approach induces medialisation of the centre of rotation (COR) and a lowering of the humerus, leading to an increase of the deltoid-moment arm, which restored 
good elevation in pseudoparalytic shoulders. This Grammont design yields satisfactory functional results, but numerous publications demonstrated that this improvement in elevation comes at a price $[2,13,27,29,30,33,38,40,43,44,48]$ : loss of medial and lateral rotation [23, 25], loss of shoulder contour, risk of instability and a high percentage of scapular notching. The latter complication is reported in around $75 \%$ of cases $[2,15,43]$ and up to $96 \%$ in some series [48], and it can be observed as early as three to six months postoperatively [34, 40, 43]. It has been demonstrated that this notching is due to friction between the polyethylene humeral bearing and the pillar of the scapula [11], leading to the additional risk of creating a foreign body reaction due to polyethylene debris [34]. Half of the scapular notches are evolutive and are responsible for decreased function and greater risk of glenoid loosening [37]. To avoid this complication, many solutions have been proposed: bony lateralisation [3]; different techniques of baseplate implantation, such as a lower implantation, flush to the inferior rim of the glenoid cavity $[4,38]$ and/or applying an inferior tilt [22, 23, 26, 31]; modification of the shape of the glenosphere (eccentric [49] or oversized [10, 49]); modification of the humeral-neck-shaft angle [33]. Some authors chose metallic lateralisation of the COR, which keeps the humeral bearing away from the pillar of the scapula. Several implants were designed according to this concept: the RSP (DJO Surgical, Austin, TX, USA) [16], the SMR (Lima, Udine, Italy) [5-7] and the Arrow RSA (FH Orthopedics, Mulhouse, France) [28, 42]. In the latter, the design of the glenosphere and metal baseplate allows COR lateralisation of $8.5 \mathrm{~mm}$. The metaphyseal part of the humeral stem has a fixed angle of $135^{\circ}$, and the polyethylene cup increases this angle to a final $155^{\circ}$. This creates an extra lateralisation of $4 \mathrm{~mm}$ of the humerus when compared with a Delta-type humeral stem [27]. Moreover, the polyethylene humeral bearing is deeper, thus decreasing the risk of instability, and has a predesigned medial notch. We hypothesised that this lateralised design would lead to a decreased incidence of scapular notching.

The goal of this study was to analyse a series of Arrow RSAs to determine the incidence of scapular notching, potential risk factors for scapular notching and associated outcomes.

\section{Materials and methods}

\section{Patient selection}

No ethical approval was required from the different institutions involved in this continuous series.

A retrospective review was conducted of a computerised database that contains files of all patients who had shoulder arthroplasty performed by a surgeon in our group. All patients who underwent primary RSA between October 2003 and
October 2012 for pseudoparalytic shoulder due to massive irreparable rotator cuff tear (associated or not with arthritis) were included. Patients were excluded if they had posttraumatic arthritis, primary osteoarthritis with no rotator cuff tear, rheumatoid arthritis, chronic dislocation, a tumor or had undergone revision surgery. Minimum follow-up was two years, and all patients were retrospectively reviewed by their surgeon, or by the first author (DK) specifically, for this study during 2014. Patients who were not available for follow-up in 2014 were excluded.

One hundred and thirty four patients (100 women, 34 men) underwent 140 RSAs for massive irreparable rotator cuff tear and a pseudoparalytic shoulder. Mean patient age was 72 years [range 52-90, standard deviation (SD) 6,91]. One hundred patients also had arthritis with upward migration of the humeral head (cuff-tear arthropathy), except for two patients who presented with arthritis without any narrowing of the subacromial space. Thirty-eight patients presented an upward migration of the humeral head but no arthritis. Twenty-six patients had already undergone surgical rotator cuff repair. The right shoulder was involved one in 94 cases and the left in 46 cases. In 97 cases, the operated arm was dominant.

\section{Pre-operative clinical evaluation}

All patients had a pre-operative examination to evaluate level of pain, function and physical findings including range of motion (ROM). Pain was evaluated using the ConstantMurley score [9]; the Simple Shoulder Test (SST) [32], Subjective Shoulder Value (SSV) [17] and body mass index (BMI) were recorded. Pain was rated as severe, with a mean of $3 / 15$ (range $0-12$ ). Activities of daily living were impaired (6, $7 / 20$, range $1-16$, SD 2,9) and active ROM was limited. Mean active flexion was $73^{\circ}$ (range 10-160, SD 32), mean external rotation at $90^{\circ}$ shoulder abduction was $30^{\circ}$ (range $0-90, \mathrm{SD}$ 26), mean Constant-Murley score 26 and mean weighted Constant score $36 \%$.

\section{Pre-operative radiological evaluation}

Radiologic evaluation included standard anteroposterior, lateral and axillary views of the shoulder joint and an arthrocomputed tomography (CT) scan to assess glenoid bone stock, deltoid muscle and remaining cuff quality, rotator cuff-tear size and extent of its retraction according to the Patte classification [35]. Fatty degeneration was assessed according to the Goutallier classification [18]. Pre-operative radiographic examination revealed six Hamada grade 2, 33 grade 3, 73 grade 4 (51 type 4a, 22 type $4 b$ ) and three grade 5 . For 23 patients, the Hamada classification was not available; the Walch classification [46] was available for 64 cases. Fifty-five (86 \%) were type A, 42 type A1 (66 \%) and 13 type A2. Nine were classified as type B (5 B1, 4 B2) and two as type C. 
All patients had complete rupture of the supraspinatus: Patte classification stage 1 in one, stage 2 in seven and stage 3 in 111. The proximal stump of the tendon was more medial than the glenoid in $11(n=130)$. Fatty degeneration of the supraspinatus was grade 1 in one patient, grade 2 in seven, grade 3 in 70 and grade 4 in $38(n=116)$. The infraspinatus tendon was not torn in five patients. When it was torn, the tendon was retracted to the bony insertion in nine, to the level of the humeral head in 23 and to the glenoid in 84; it was more medial than the glenoid border in ten $(n=131)$. Fatty degeneration of the infraspinatus was grade 1 in seven patients, grade 2 in 17, grade 3 in 68 and grade 4 in $24(n=116)$. Nighty-nine patients had a subscapularis tendon tear, mostly at the level of its superior third $(n=47)$; the tendon was retracted to at least half of the humeral head in $44(n=132)$. Fourteen cases had no fatty degeneration of the subscapularis tendon, 38 hade grade 1, 25 had grade 2, 33 had grade 3 and five had grade 4. Data for fatty infiltration of the subscapularis was not available for 24 patients. The Favard classification was not available for this study.

\section{Operative data}

All patients were operated using the Arrow universal shoulder arthroplasty (FH Orthopedics) by one of the four senior authors (DK, JK, PV, KE) in four different centres. Modification of the implant design occurred during this ten year study. Because there were some cases of dissociation between the humeral polyethylene bearing and the humeral stem, a metallic tray was added to the humeral bearing in 2007 . Arthroplasties were performed through a superior approach in 115 cases and a deltopectoral approach in 25 . The biceps tendon was intact in three cases, dislocated in 15 , degenerative in 49 and torn in $59(n=128)$. Tenodesis of the long head of the biceps was systematically performed in the bicipital grove in the 67 cases in which the tendon was not already disrupted. After extensive resection of the subacromial bursa and degenerative fibrous tissues, sectioning the superior third of the subscapularis allowed lowering of the humeral head when the joint was stiff. Particular attention was paid to expose the glenoid with a circumferential periglenoidal capsulectomy, especially when a superior approach was used. This allowed good inferior and posterior translation of the humerus. The humeral head was cut with $10-20^{\circ}$ of retroversion. Fixation of the humeral stem was ensured either by cementing or with a press-fit technique depending on surgeon discretion. The stem was press-fitted in 106 cases (76\%), in which the metaphysis was meticulously grafted with cancellous bone from the humeral head before implant impaction; 34 cases were cemented. We attempted to place the metal-backed glenoid implant flush to the inferior border of the glenoid cavity with a slight inferior tilt.
The shoulder was tested intra-operatively, especially in axial rotations, to detect any eventual impingement between the humeral bearing and the pillar of the scapula. Because of implant lateralisation, an acromioplasty and/or a tuberoplasty was often necessary to avoid any impingement between the greater tuberosity and the acromion during anterior flexion. The humeral stem was size 8 in 26 cases, 10 in 68 cases, 12 in 41 cases and 14 in three cases. The size of glenoid metal-backed baseplate was 44 in 110 cases $(79 \%)$ and 46 in 30 cases; glenosphere size was 36 in $83 \%$ of cases. Humeral bearing thickness was always $0 ; 54$ bearings were full polyethylene (first-generation design before 2007), and the remaining 86 had a metallic tray.

\section{Post-operative clinical and radiographic evaluation}

Patients were systematically reviewed every year by their surgeon. Those who had no clinical evaluation in 2014 were asked to return for clinical assessment by one of the senior authors (DK). At the latest consultation, patients were evaluated to assess level of pain, function and ROM using the Constant-Murley score, SST, visual analogue scale (VAS) and SSV. Subjective satisfaction was assessed by asking patients at follow-up how they felt compared with before surgery and was graded using a 4 -point scale: $1=$ much better; $2=$ better; $3=$ same; $4=$ worse.

Three standardised views of the shoulder were used for post-operative radiographic analysis in standard anteroposterior, lateral and axillary views. These were analysed to determine the presence of scapular notching according to the Nerot-Sirveaux classification system; to measure baseplate position (low, centre, high) and tilt (superior, inferior, none); ratio between lateral offset of the operated and healthy control side; and importance of arm lengthening by measuring the distance between the top of the humeral head and the inferior cortex of the acromion.

\section{Statistical analysis}

Student's $t$ test was used for statistical analysis when two groups were compared: e.g. group 1 with no scapular notch vs group 2 with scapular notch. When comparison involved more than two groups, analysis of variance (ANOVA) was used. The level of significance was set at $p=0.05$.

\section{Results}

Clinical and radiological results of a patient in this study 


\section{Clinical results}

At a mean follow-up of 45 months (range 24-120, SD 20), pain, function and satisfaction were all significantly improved. Pain levels improved from 3 (range 0-12) to 13,7 (range 5-15, $p<0.0001)$. Evaluation of the activities of daily living improved from 6.7 (range;1-16) to 15,5 (range $5-20, p<$ $0.0001)$. ROM was significantly improved in a clinically important and statistically significant fashion. Mean active flexion increased from $73^{\circ}$ (range $10-160^{\circ}$ ) to $132^{\circ}$ (range 40 $180^{\circ}, p<0.0001$ ). Mean active abduction increased from $61^{\circ}$ (range $20-150^{\circ}$ ) to $108^{\circ}$ (range $40-170^{\circ}, p<0.0001$ ). Mean external elbow rotation improved from $20^{\circ}$ (range $0-70^{\circ}$ ) to $29^{\circ}$ (range $0-70^{\circ}, p=0.0003$ ). Mean external rotation at $90^{\circ}$ shoulder abduction improved from $30^{\circ}$ (range $0-90^{\circ}$ ) to $54^{\circ}$ (range $0-100^{\circ}, p<0.0001$ ). Strength increased from 1,4 (range $0-10)$ to 6 (range $0-16, p<0.0001$ ). The Constant score improved from 26 (range 11-53) to 64 (range 26-85, $p<0.0001$ ), and the weighted Constant improved from $36 \%$ (range 15 75 ) to $92 \%$ (range $41-123, p=0.0001$ ).

At the time of latest follow-up, mean SST was 8.66 yes ( $n=$ 114).; $91 \%$ of patients felt they were better or much better than pre-operatively, and seven (5\%) felt they were worse. In the latter seven, an obvious cause could be found in five, two had deltoid palsy, one had polyarticular osteoarthritis (spine, hips, knees), one had an intraoperative glenoid fracture and one had glenoid loosening).

\section{Radiographic analysis}

At the time of latest follow-up, 41 patients (29\%) presented with a scapular notch: 20 grade 1, 18 grade 2 and three grade 3. The latter three had a follow-up of 44, 70 and 84 months, respectively. During the final two years of follow-up, notching did not evoluate, and there was no clinical consequence for two of them. The third one had humeral stem subsidence, which has not been revised so far.

\section{Complications}

Complications occurred in 30 patients ( $22 \%)$. One patient had an intra-operative fracture of the humeral shaft, which was treated with open reduction and internal fixation (ORIF) and healed uneventfully. Two patients had an intra-operative glenoid fracture. The glenoid baseplate of the implant has an anterior winglet, which was sufficient to stabilise the fracture. Both fractures healed uneventfully. One patient had a traumatic fracture of the greater tuberosity at three months, which was treated non-operatively and healed uneventfully. One patient had an acromial fracture, which was successfully managed non-operatively. Four patients had transient brachial plexus or axillary nerve palsy. Six cases of dissociation of the humeral bearing occurred with the first-generation implant, which ultimately led to design modification in 2007. Those six cases were revised by simply changing the polyethylene humeral bearing. as the implants were well fixed. Two patients had wear of the humeral bearing: one necessitated revision with a simple change of the bearing. Three patients had loosening and subsidence of the uncemented humeral; one had to be revised, and another one had progressive varus of the stem with no clinical impact $\left(130^{\circ}\right.$ of active elevation, no pain, Constant score 63). Four patients had glenoid implant loosening, one of which had to undergo revision. There were three cases of stiffness and three of post-operative infection, which were all revised.

\section{Revision operations}

Twelve patients in whom the device failed required revision $(8.9 \%)$. Seven shoulders required only a simple change of the humeral bearing ( 6 for dissociation, 1 for wear). One patient with glenoid loosening and another with humeral loosening were both revised successfully to another RSA. Three infections required revision: two underwent a one-stage procedure with immediate reimplantation followed by three months of antibiotherapy (1 Propionibacter acnes and one Staphylococcus aureus); the third underwent simple debridement and cleaning followed by antibiotherapy (P. acnes). Excluding cases of dissociation due to an old implant design, the complication rate was $17.7 \%$ and the revision rate $4.4 \%$.

\section{Analysis of occurrence of scapular notch}

The cohort was divided in two groups: group 1, who did not develop a scapular notch; and group 2, who presented a scapular notch at the last follow-up.

Group 1: 99 arthroplasties in 95 patients (70 women, 25 men).

Group 2: 41 arthroplasties in 40 patients (30 women, 10 men).

Pre-operative results in the two groups are summarised in Table 1. Patients in group 2 had a greater pre-operative active ROM and higher Constant-Murley score. This, however, only reached statistical significance for active flexion $(p=0,01)$ and abduction $(p=0,003)$.

There were no differences between groups regarding gender, operated side, underlying diagnosis, Walch classification or type of humeral stem fixation. There was a significantly greater number of scapular notches in patients operated on the dominant side: $64 \%$ in group 1 versus $83 \%$ in group 2 $(p=0,04)$. Patients in group 2 presented preoperatively with significantly more-advanced cuff-tear arthropathy according to the Hamada classification, with $74 \%$ of shoulders classified as $4 \mathrm{a}$ and $4 \mathrm{~b}$ in group 2 versus $58 \%$ in group $1(p=0,004)$. 
Table 1 Pre-operative clinical findings in the two groups: no significant difference except for active flexion $(p=0,016)$ and abduction $(p=0,003)$

\begin{tabular}{|c|c|c|c|c|c|c|c|c|c|}
\hline Preop & Pain & $\mathrm{ADL}$ & Active flexion & Active abduction & ER1 & ER2 & IR cst & Strength & Cst score \\
\hline Group 1: no notch & $\begin{array}{l}3.2 \\
(0-15) \\
\text { SD } 3.7\end{array}$ & $\begin{array}{l}6.5 \\
(1-14) \\
\text { SD 2.7 }\end{array}$ & $\begin{array}{l}68^{\circ} \\
(10-160) \\
\text { SD } 32\end{array}$ & $\begin{array}{l}56^{\circ} \\
(20-120) \\
\text { SD } 25\end{array}$ & $\begin{array}{l}21^{\circ} \\
(0-70) \\
\text { SD } 14\end{array}$ & $\begin{array}{l}31^{\circ} \\
(0-90) \\
\text { SD } 26\end{array}$ & $\begin{array}{l}4,9 \\
(0-10) \\
\text { SD } 2.7\end{array}$ & $\begin{array}{l}1.3 \\
(0-10) \\
\text { SD 2.2 }\end{array}$ & $\begin{array}{l}25.2(36 \% \\
(2-50) \\
\text { SD } 9.3\end{array}$ \\
\hline Group 2: notch & $\begin{array}{l}2,8 \\
(0-10) \\
\text { SD } 3.4\end{array}$ & $\begin{array}{l}7.2 \\
(2-16) \\
\text { SD } 3.3\end{array}$ & $\begin{array}{l}83^{\circ} \\
(30-140) \\
\text { SD 28 }\end{array}$ & $\begin{array}{l}73^{\circ} \\
(30-150) \\
\text { SD } 29\end{array}$ & $\begin{array}{l}16^{\circ} \\
(0-50) \\
\text { SD } 12\end{array}$ & $\begin{array}{l}27^{\circ} \\
(0-90) \\
\text { SD 25 }\end{array}$ & $\begin{array}{l}4.7 \\
(0-10) \\
\text { SD 2.6 }\end{array}$ & $\begin{array}{l}1.5 \\
(0-8) \\
\text { SD 2.2 }\end{array}$ & $\begin{array}{l}27,9(38 \% \\
9-53 \\
\text { SD } 12.5\end{array}$ \\
\hline
\end{tabular}

Preop pre-operative, $S D$ standard deviation, $A D L$ activities of daily living, $E R, I R, C s t$

There were more shoulders classified $4 \mathrm{~b}$ in group 2 than in group $1(p=0,001): 46 \%$ type $4 \mathrm{a}$ and $12 \%$ type $4 \mathrm{~b}$ in group 1 versus $35 \%$ and $39 \%$ in group 2 . Moreover, $36 \%$ in group 1 were classified type 3 versus $0 \%$ in group 2 . Patients in both groups were significantly improved after surgery, and there was no significant difference in pain, ROM or Constant score between groups at the last follow-up. These results are summarised in Table 2 .

We attempted to determine whether cases with final flexion $<110^{\circ}$ were more frequent in group 2 and, if so, then why. There were 17 cases (17\%) in group 1 at the last follow-up and we found an explanation for ten: one intra-operative humeral shaft fracture, one infection, one Parkinson's disease, one pre-operative ankylosis, one humeral loosening, one glenoid loosening, one axillary palsy and one acromial fracture. In group 2 there were five cases $(12 \%)$ and an explanation was found in two: one humeral loosening with humeral stem subsidence, and one glenoid loosening. The difference between groups did not reach significance. The ratio between baseplate and glenosphere size was compared to determine whether the association of a small glenosphere with a large baseplate could lead to mechanical impingement with the humeral bearing, which could increase the risk of scapular notching. This ratio was not found to be significantly different between groups. Nine cases had a bony scapular spur at the last follow-up: three $(3 \%)$ in group 1 and six $(14,6 \%)$ in group 2. A spur occurred significantly more frequently in group $2(p=0,003)$. Obesity was found to decrease the risk of developing a scapular notch, with incidence increasing when BMI was <30. BMI values were available for 59 patients (35/99 in group $1 ; 27 / 41$ in group 2): 49 had a BMI $<30$, of whom 21 presented a scapular notch (43\%); three of ten patients with a BMI $>30$ had a scapular notch $(30 \%)$. However, this difference did not reach statistical significance $(p=0,09)$. Glenoid baseplate position had a significant influence on the development of a scapular notch. This criteria was analysed in 123 cases: 108 baseplates $(87,80 \%)$ were classified as being centred or implanted in a low position. Among them, 32,5\% developed a scapular notch. Fifteen $(12,20 \%)$ were implanted high, and nine of them $(60 \%)$ presented a scapular notch. This difference was significant $(p=0,0033)$. In relation to baseplate tilt, the rate of scapular notching was be significantly higher $(p=0.0029)$ when tilted superiorly than when tilted inferiorly (100\% vs $26 \%$ ). There was no difference $(p=0,91)$ in lateral offset between groups: in group 2 , the difference between the operated and healthy control side was $-0,41 \mathrm{~cm}(n=28)$ versus $-0,44 \mathrm{~cm}$ in group $1(n=65)$. No statistical difference was found in the degree of humeral lowering between groups, and mean subacromial space was $4 \mathrm{~cm}$ in group $2(n=27)$ versus $3,85 \mathrm{~cm}$ in group $1(n=65)$. Glenoid baseplate implantation for the three cases with grade 3 scapular notch was centred in two and low in one (Fig. 1). Two cases had good functional results despite a superior tilt in one with a VAS $=0$, an SSV at $90 \%$ and $100 \%$, respectively, and a weighted Constant-Murley score $>90 \%$ : 63 (91\%) for the first and $65(94 \%)$ for the second. The case with a grade 3 scapular notch had a centred glenoid baseplate with a superior tilt. Clinically, the patient had no pain but a poor ROM and a weighted Constant score of $41 \%$. This was the patient with unrevised humeral subsidence who refused revision surgery.

Table 2 Post-operative clinical findings: no significant differences between groups

\begin{tabular}{llllllllll}
\hline Postop & Pain & ADL & Active flexion & Active abduction & ER1 & ER2 & $\begin{array}{l}\text { IR } \\
\text { cst }\end{array}$ & $\begin{array}{c}\text { Strength } \\
\text { Cst } \\
\text { score }\end{array}$ \\
\hline Group 1: no notch & 13,7 & 15.1 & $132^{\circ}$ & $108^{\circ}$ & $30^{\circ}$ & $54^{\circ}$ & 6.4 & 6.4 \\
& $(5-15)$ & $(6-20)$ & $(40-180)$ & $(40-170)$ & $(0-70)$ & $(0-95)$ & $\begin{array}{l}(2-10) \\
(0-16)\end{array}(35-83)$ \\
& SD 2.8 & SD 3.5 & SD 27 & SD 25 & SD 17 & SD 24 & SD 2,1 & SD 3.5 & SD 9.8 \\
Group 2: Notch & 13.7 & 16.2 & $130^{\circ}$ & $109^{\circ}$ & $27^{\circ}$ & $54^{\circ}$ & 7.0 & 5.6 & $64(93 \%)$ \\
& $(10-15)$ & $(5-20)$ & $(40-160)$ & $(80-160)$ & $(0-70)$ & $(10-100)$ & $(2-10)$ & $(0-13)$ & $(26-85)$ \\
& SD 2.1 & SD 3.8 & SD 25 & SD 25 & SD 17 & SD 23 & SD 2.4 & SD 3.0 & SD 10.5 \\
\hline
\end{tabular}


Fig. 1 Grade 3 scapular notch. a Standard anteroposterior radiograph of the left/right shoulder at 12 months. b At 55 months, the scapular notch has not evolved. Densification at the bottom of the notch it is not going to evolve. Despite a superior tilt of the baseplate, this patient had good functional result at the latest follow-up, with a ConstantMurley score of 63 (90\%), no pain, and a satisfactory active range of motion. Active flexion was $130^{\circ}$, active external rotation $90^{\circ}$, shoulder abduction $60^{\circ}$ and internal rotation to the level of $\mathrm{T} 12$
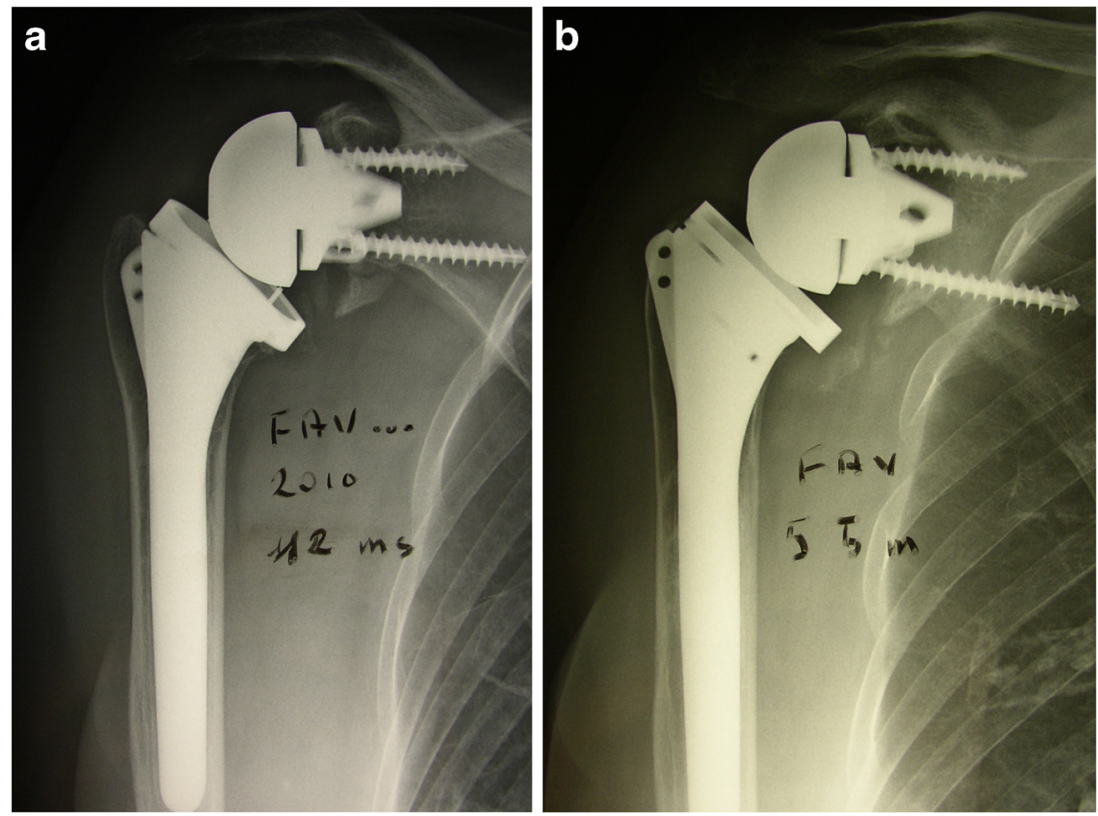

\section{Discussion}

In a previous study, we published a preliminary report regarding our results with the Arrow RSA, which creates a metallic lateralisation of the COR, particularly concerning scapular notching [42]. In that study, no scapular notching was found. The study reported here, using the same implant with an extended follow-up and a greater number of patients, found a higher incidence of scapular notching, which reached $29 \%$.

The incidence of scapular notching has previously been reported with Grammont-type implants (Delta III, DePuy, Warsaw, IN, USA). This implant has a more medialised design, with a COR at the bone-implant interface. Rates of scapular notching varied between $40 \%$ and $74 \%[2,12,13$, $15,36,40,43,44]$, with a maximum of $96 \%$ [48]. In most publications, the rate of scapular notching continued to increase over time.

We identified several risk factors for scapular notching. In our series, patients who had a better pre-operative ROM and therefore a greater post-operative ROM were more at risk for developing a scapular notch [30]. This was also true for patients with a $\mathrm{BMI}<30$, which is in agreement with previous studies [14, 29]. Except for a lower risk of notching in obese patients, BMI did not influence the rate of complications in our series as opposed to results reported by Gupta et al. [21]. However, as opposed to reports in previous publications [30, 38,40 ], we found no influence of scapular notching on postoperative active ROM or on complication rates; however, as with Favard et al. [15], our results confirmed the deleterious effect of superior tilt.

Lateralisation of the COR leads to a theoretical risk of increasing the shear forces applied on the glenoid [3]. This, however, did not lead to an increase in glenoid loosening in our study. Two of the four glenoid loosenings observed in our series were traumatic, occurring as a result of a fall in the early post-operative period. Radiographic analysis of a third case of loosening, which occurred at two years from the index procedure, showed that primary fixation had never been achieved. Therefore, it seems that — as opposed to some literature reports $[1,3,36]$ - glenoid loosenings in our series were not due to scapular notching and subsequent progressive glenoid destabilisation. Special attention must be paid to primary fixation of the glenoid baseplate, eventually adding a bone graft and/or using a long-peg glenoid implant. Frankle et al. [16] reported $12 \%$ of glenoid loosening of a first-generation Encore RSA. This implant glenosphere is two thirds of a sphere, whereas that of the Arrow RSA is only half a sphere. This probably contributes to the low rate of glenoid loosening found in our study. As with Harman et al. [24], we believe that good primary fixation and meticulous preparation of the glenoid bone reduces the risk of loosening, even with a lateralised COR.

This new study confirmed that the use of a lateralised implant provided better results in axial rotation (Fig. 2) than those previously reported with a Grammont-type RSA, with a mean improvement in external rotation of $20^{\circ}$ versus $<10^{\circ}$ $[2,40,47,48]$. This allows better activities of daily leaving $[2$, 31]. Two main theories have been proposed to explain the improvement in external rotation: lateralised design of the implant, and position of the glenoid baseplate. Indeed, an increase in lateral offset with a lateralised implant could restore external rotator tension. On the other hand, an inferior tilt, implantation of the baseplate flush with the inferior glenoid rim [4] or the use of a larger glenosphere can limit friction between the humeral cup and the scapular pillar. The absence of friction between humerus and pillar could also reduce 

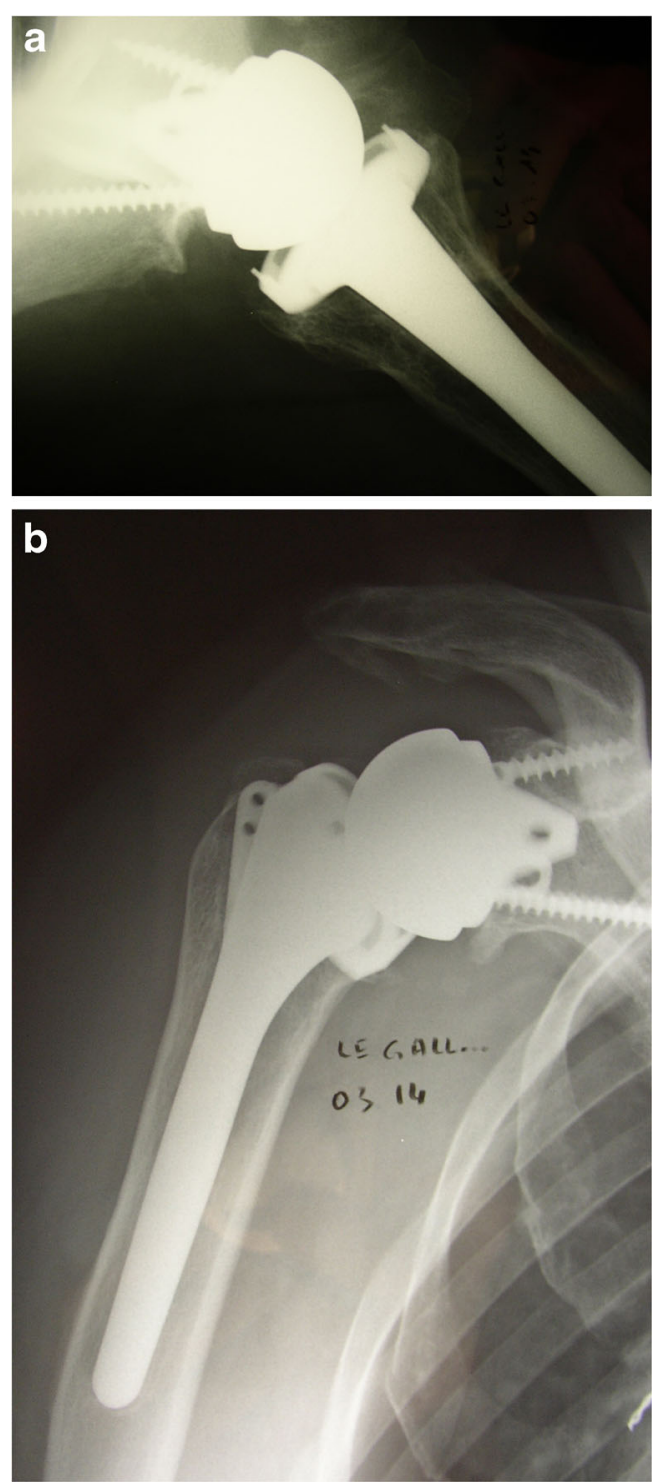

Fig. 2 Clinical and radiological results for a 79-year-old patient at final follow-up of 60 months. Active range of motion was very satisfactory, especially in axial rotation. Position of the glenoid baseplate is ideal. a-d Active range of motion. e Final anteroposterior shoulder radiograph

scapular notching [50]. Edwards et al. [13], in a randomised study, found that inferior glenoid tilt did not decrease scapular notching. Moreover, it has also been suggested that too much inferior tilt can increase scapular notching [31,39] because of the induced humeral bearing medialisation. However, our findings showed that the risk of scapular notching was significantly higher when the glenoid was not inferiorly tilted.

It has been advocated in the litterature $[6,7]$ that with increased lateralisation, the final active elevation would be decreased. This was not confirmed in our study, in which active elevation was comparable with the DELTA-type RSA. Lateralisation can be obtained by lateralising the COR and also by lateralising the humerus and increasing the distance between the humeral bearing and the scapular pillar. Several authors [33, 45], such as the designers of the Trabecular Metal Reverse Shoulder System (Zimmer, Warsaw, IN, USa) suggested decreasing the humeral cut angle from the $155^{\circ}$ of the Grammont implant in order to lateralise the humerus; this is known to increase the risk of instability [16]. Rates of postoperative instability after Grammont-type RSA vary between $3 \%$ and $9 \%[2,47,48]$. No post-operative instability was found in our series, probably partly due to the fact that the humeral cup of the Arrow is deeper. Clark et al. [8], in a study of 120 lateralised RSA (RSP, DonJoy Surgical, Austin, TX, USA) emphasised the stabilising effect of lateralisation due to an increase in compressive forces.

An option other than metallic for lateralising the COR in order to reduce scapular notching has been proposed by Boileau et al. [3]: the bony increased-offset RSA (BIORSA). By adding a bone graft between the glenoid and baseplate, a long-necked scapula is created that lateralises the COR while maintaining it at the bone-implant interface. This was supposed to provide the advantages of lateralisation, including a lower risk of scapular notching, while decreasing the shear forces applied on the glenoid component. However, the latest reports still show grade 3 or 4 evolutive scapular notches [3, 41]. Another way to avoid mechanical impingement between the polyethylene humeral bearing and the scapular pillar is to predesign a notch on the humeral bearing, as in the Arrow RSA [4].

This study has several limitations. Firstly, we were not able to provide accurate measurement of glenoid baseplate tilt. We believe, as do Favard et al. [15], that it is very difficult to reproduce good radiographs in a standardised manner that allows precise measurements, such as the glenoscapular angle. Secondly, as with Boileau et al. [3], we were unable to measure posterior notching, which seems to be more frequent than commonly described (Fig. 3).

The best way to avoid posterior impingement between the polyethylene humeral bearing and the pillar is probably intraoperative testing. When de-coaptation of the joint occurs in external rotation, care must be taken to check for the presence of posterior osteophytes on the glenoid or to increase glenosphere retroversion or size. Boughrebi et al. [4] suggested a more posterior predesigned notch. Another bias in studies of scapular notching is that different classifications of scapular notches are imprecise due to the variable direction of inferior screws among different prosthetic designs, or even in the same arthroplastic design where nonlocking screws can have different directions [50]. This changes the distance between the inferior screw and the scapular pillar; therefore, we feel that the best way to determine whether a notch is benign and nonevolutive is the presence of densification at the bottom of the notch.

Although this was a multicentric study, which implies it has the limitations of a multicentric study, all surgeons are designers of the RSA used, and all patients were operated 
Fig. 3 Posterior notch not visible on anteroposterior view. As in Fig. 1, densification at the bottom of the posterior notch can be observed
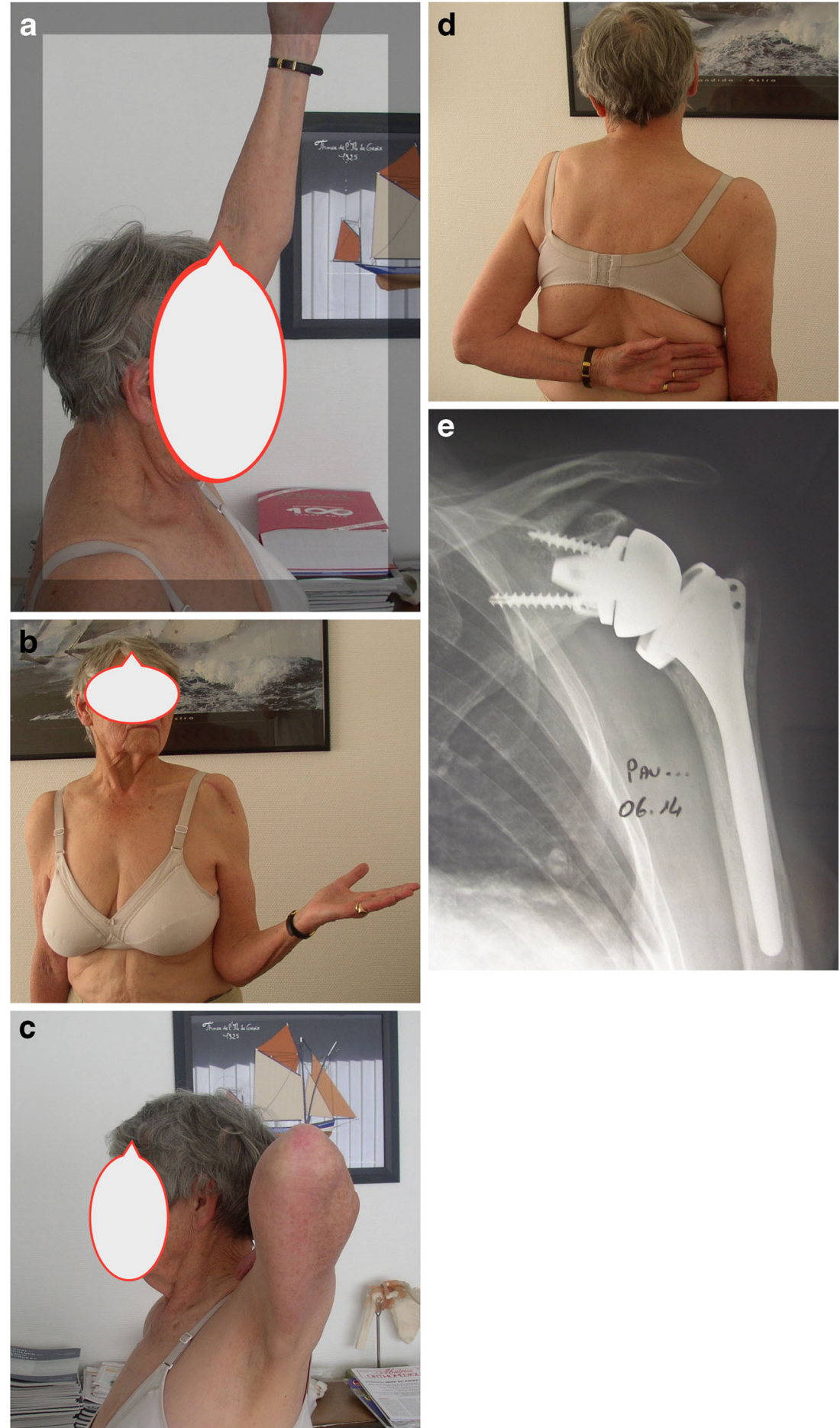

following a standardised technique with a standardised rehabilitation protocol. The study was also retrospective, with a follow-up from 24 to 120 months. During this lengthy period, operative technique changed, taking into account the most recent recommendations such as low glenoid implantation and inferior tilt. To date, we have found no statistically significant difference between the rate of scapular notching during this entire study period.

\section{Conclusion}

In conclusion, the Arrow RSA still has a $29 \%$ rate of scapular notching, with no evolutive notches at $>$ two years of follow-up. This rate is less than with more medialised implants. We identified several risk factors that can lead to the development of scapular notching in RSA: patients with a good pre-operative $\mathrm{ROM}$ and patients with a $\mathrm{BMI}<30$ are more likely to develop 
a scapular notch. A superior tilt and a high baseplate implantation are also significant risk factors for notching. However, grades 1 and 2 notches in our study had no influence on functional outcome.

The design of the arthoplastic system of course plays a role in the incidence of scapular notching. It appears that a lateralised implant with metallic lateralisation of the COR, lateralisation in the humerus with a humeral cut at $135^{\circ}$, a humeral bearing at $155^{\circ}$ and a predesigned notch on the humeral bearing - such as in the Arrow RSA - can provide a low rate of scapular notching. The design must also ensure good primary baseplate fixation.

Surgical technique is also of great importance, ensuring low baseplate fixation and a slight inferior tilt. Great care must be taken during intraoperative testing to detect any mechanical impingement between humerus and scapular pillar, especially in axial rotation and elevation.

Conflict of interest Jean Kany, Denis Katz, Philippe Valenti and Kamil Elkholti are consultants for FH Orthopedics (Mulhouse, France) and have received royalties for a product related to this work. This might pose a conflict of interest in connection with the submitted article.

Ethics Each author certifies that all investigations were conducted in conformity with ethical principles of research and that informed consent for participation in the study was obtained.

Open Access This article is distributed under the terms of the Creative Commons Attribution 4.0 International License (http:// creativecommons.org/licenses/by/4.0/), which permits unrestricted use, distribution, and reproduction in any medium, provided you give appropriate credit to the original author(s) and the source, provide a link to the Creative Commons license, and indicate if changes were made.

\section{References}

1. Berliner JL, Regalado-Magdos A, Ma B, Feeley BT (2015) Biomechanics of reverse total shoulder arthroplasty. J Shoulder Elbow Surg 24(1):150-160. doi:10.1016/j.jse.2014.08.003

2. Boileau P, Watkinson DJ, Hatzidakis AM, Balg F (2005) Grammont reverse prosthesis: design rationale and biomechanics. J Shoulder Elbow Surg 14:147S-161S. doi:10.1016/j.jse.2004.10. 006

3. Boileau P, Moineau G, Roussanne Y, O'Shea K (2011) Bony increased offset reversed shoulder arthroplasty: minimizing scapular impingement while maximizing glenoid fixation. Clin Orthop Relat Res 469(9):2558-2567. doi:10.1007/s11999-011-1775-4

4. Boughebri O, Duparc F, Adam JM, Valenti P (2011) Arthroscopic dynamic analysis of scapular notching in reverse shoulder arthroplasty. Orthop Traumatol Surg Res 97(8):779-784. doi:10. 1016/j.otsr.2011.07.014

5. Castagna A, Delcogliano M, De Caro F, Zivzeri G, Borroni M, Gumina S et al (2013) Conversion of shoulder arthroplasty to reverse impalnts: clinical and radiological rtesults using a modular system. Int Orthop (SICOT) 37(7):1297-1305

6. Castagna A, Randelli M, Garofalo R, Maradei L, Giardella A, Borroni M (2010) Mid-term results of a metal-backed glenoid component in total shoulder replacement. J Bone Joint Surg (Br) 92(10): 1410-1415

7. Chisholm C, Poon PC (2012) An in vivo kinematics study of the reverse shoulder joint replacement. Eur J Orthop Surg Traumatol 22(8):655-660. doi:10.1007/sOO590-011-0901-Z

8. Clark JC, Ritchie J, Song FS, Kissenberg MJ, Tolan SJ, Hart ND et al (2012) Complication rates, dislocation, pain, and post operative range of mobility after reverse shoulder arthroplasty in patients with and without repair of the subscapularis. J Should Elbow Surg 21(1):36-41. doi:10.1016/j.jse.2011.04.009

9. Constant CR, Murley AH (1987) A clinical method of functional assessment of the shoulder. Clin Orthop Relat Res 214:160-164

10. Cuff D, Pupello D, Virani N, Levy J, Frankle M (2008) Reverse shoulder arthroplasty for the treatment of rotator cuff deficiency. J Bone Joint Surg Am 90(6):1244-1251. doi:10.2106/JBJS.G.00775

11. Day JS, MacDonald DW, Olsen M, Getz C, Williams GR, Kurtz SM (2012) Polyethylene wear in retrieved reverse total shoulder components. J Should Elbow Surg 21(5):667-674. doi:10.1016/j. jse.2011.03012

12. Delloye C, Joris D, Colette A, Eudier A, Dubuc JE (2002) Mechanical complications of total shoulder inverted prosthesis. Rev Chir Orthop 88(4):410-414

13. Edwards TB, Trappey GJ, Riley C, O'Connor DP, Elkousy HA, Garstman GM (2012) Inferior tilt of the glenoid component does not decrease scapular notching in reverse shoulder arthoplasty: results of a prospective randomized study. J Should Elbow Surg 21(5):641-646. doi:10.1016/j.jse.2011.08.057

14. Falaise V, Levigne C, Favard L (2011) Scapular notching in reverse shoulder arthroplasty: the influence of glenometaphyseal angle. Orthop Trauma Tol Surg Res 97(6 Suppl):S131-S137

15. Favard L, Lautmann S, Sirveaux F, Oudet D, Kerjean Y, Huguet D (2000) Hemiarthroplasty versus reverse arthroplasty in the treatment of osteoarthritis with massive rotator cuff tear. In: Walch G, Boileau P, Mole D (eds) Shoulder Prostheses. Two to Ten Years Follow-up. Sauramps Medical, Paris, pp 261-268

16. Frankle M, Siegal S, Pupello D, Saleem M, Vasey M (2005) The Reverse shoulder prosthesis for glenohumeral arthritis associated with severe rotator cuff deficiency. A minimum two-year followup study of 60 patients. J Bone Joint Surg Am 87(8):1697-1705

17. Gilbart MK, Gerber C (2007) Comparison of the sujective shoulder value and the Constant score. J Should Elbow Surg 16(6):717-721. doi:10.1016/j.jse.2007.02.123

18. Goutallier D, Postel JM, Bernageau J, Lavau L, Voisin MC (1994) Fatty muscle degeneration in cuff ruptures. Pre- and postoperative evaluation by CT scan. Clin Orthop Relat Res (304):78-83

19. Grammont PM, Trouilloud P, Laffay JP, Deries X (1987) Etude et réalisation d'une nouvelle prothèse d'épaule. Rhumatologie 39: 407-418

20. Grammont PM, Baulot E (1993) Delta shoulder prosthesis for rotator cuff rupture. Orthopedics 16(1):65-68

21. Gupta AK, Chalmers PN, Rahman Z, Bruce B, Harris JD, McCormick $F$ et al (2014) Reverse total shoulder arthroplasty in patients of varying body mass index. J Should Elbow Surg 23(1): 35-42. doi:10.1016/j.jse.2013.07.043

22. Gutierrez S, Greiwe R, Frankle M, Siegal S, Lee WE III (2007) Biomechanical comparison of component position and hardware failure in the reverse shoulder prosthesis. J Should Elbow Surg 16:S9-S12. doi:10.1016/j.jse.2005.11.008

23. Gutiérrez S, Levy JC, Lee WE, Keller TS, Maitland ME (2007) Center of rotation affects abduction range of motion of reverse shoulder arthroplasty. Clin Orthop Relat Res Number 458:78-82

24. Harman M, Frankle M, Vasey M, Banks S (2005) Initial glenoid component fixation in reversetotal shoulder arthroplasty: A biomechanical evaluation. J Should Elbow Surg 14:162S-167S. doi:10. 1016/j.jse.2004.09.030 
25. Henninger HB, Barj A, Anderson AE, Bachus KN, Burks RT, Tashjian RZ (2012) Effect of lateral offset center of rotation in reverse total shoulder arthroplasty: a biomechanical study. J Should Elbow Surg 21(9):1128-1135. doi:10.1016/j.jse.2011.07. 034

26. Jobin CM, Brown GD, Bahu MJ, Gardner TR, Bigliani LU, Levine WN et al (2012) Reverse total shoulder arthroplasty for cuff tear arthropathy: the clinical effect of deltoid lengthening and center of rotation medialization. J Should Elbow Surg 21(10):1269-1277. doi:10.1016/j.jse.2011.08.049

27. Kalouche I, Sevivas N, Wahegaonker A, Sauzieres P, Katz D, Valenti P (2009) Reverse shoulder arthroplasty: does reduced medialisation improve radiological and clinical results ?Acta Orthop. Belg Apr 75(2):158-166

28. Katz D, O'Toole G, Cogswell L, Valenti P, Sauzières P (2007) A history of the reverse shoulder prosthesis. Int J Should Surg 1:108113

29. Levigne C (2008) Scapular notching in reverse prostheses. Causes and consequences. Cahiers d'enseignement de la SOFCOT 2008 ed Elsevier-Masson: 397-406

30. Levigne C, Garret J, Boileau P, Alami G, Favard L, Walch G (2011) Scapular notching in reverse Shoulder Arthroplasty: is it important to avoid it and how ? Clin Orthop Relat Res 469(9):2512-2520. doi:10.1007/s11999-010-1695-8

31. Li X, Knutson Z, Choi D, Lobatto D, Lipman J, Craig EV et al (2013) Effects of glenosphere positioning on impingement-free internal and external rotation after reverse total shoulder arthroplasty. J Should Elbow Surg 22(6):807-813. doi:10.1016/j.jse.2012.07. 013

32. Lippitt SB, Harryman DT, Matsen FA (1993) A practical tool for evaluating function: the simple shoulder test. In: Matsen FA, FuFH HRJ (eds) The Shoulder: a balance of mobility and stability. American Academy of Orthopedic Surgeons, Rosemont, pp 501530

33. Nicholson GP, Strauss EJ, Sherman SL (2011) Scapular notching: recognition and strategies to minimize clinical impact. Clin Orthop Relat Res 469(9):2521-2530

34. Nyffeler RW, Werner CML, Simmen BR, Gerber C (2004) Analysis of a retrieved Delta III total shoulder prosthesis. J Bone Joint Surg (Br) 86B:1187-1191

35. Patte D (1990) Classification of rotator cuff lesions. Clin Orthop Relat Res 254:81-86

36. Rittmeister M, Kerschbaumer F (2001) Grammont reverse TSA in patients with Rheumatoid arthritis and nonreconstructible Rotator cuff lesions. J Should Elbow Surg 10:17-22

37. Roche CP, Stroud NJ, Martin BL, Steiler CA, Flurin PH, Wright et al (2013) The impact of scapular notching on reverse shoulder glenoid fixation. J Should Elbow Surg 22(7):963-970. doi:10.1016/ j.jse.2012.10.035
38. Sadoghi P, Leithner A, Vavken P, Hölzer A, Hochreiter J, Weber G et al (2011) Infraglenoidal scapular notching in reverse total shoulder replacement: a prospective series of 60 cases and systematic review of the literature. BMC Musculoskelet Disord 12:101. doi: 10.1186/1471-2474-12-101

39. Simovitch RW, Zumstein MA, Lohri E, Helmy N, Gerber C (2007) Predictors of scapular notching in patients managed with the Delta III reverse total shoulder replacement. J Bone Joint Surg Am 89: 588-600

40. Sirveaux F, Favard L, Oudet D, Huguet H, Walch G, Mole D (2004) Grammont inverted total shoulder arthroplasty in the treatment of glenohumeral osteoarthritis with massive rupture of the cuff. Results of a multicentre study of 80 shoulders. J Bone Joint Surg (Br) 86B:388-395

41. Terrier A, Faron A (2010) Biomechanical rationale for BIO RSA and metallic lateralization. Shoulder concept 2010. Glenoid 2010. editor. Montpellier, France: Sauramps Medical 365-70

42. Valenti P, Sauzières P, Katz D, Kalouche I, Kilinc AS (2014) Do less medialized reverse shoulder prostheses increase motion and reduce notching? Clin Orthop Relat Res 469(9):2550-2557. doi: 10.1007/s11999-011-1844-8

43. Valenti P, Boutens D, Nerot C (2001) Delta 3 reversed prosthesis for osteoarthritis with massive rotator cuff tear: Long term results $(>5$ years). In: Walch G (ed) Shoulders Prostheses. Two to Ten years follow-up. Sauramps Médical, Montpellier, pp 253-259

44. Vanhove B, Beugnies A (2004) Grammont's reverse shoulderprosthesis for rotator cuff arthropathy. A retrospective study of 32 cases. Acta Orthop Belg 70(3):219-225

45. Virani NA, Cabezas A, Gutierrez S, Santoni BD, Otto R, Frankle M (2013) Reverse shoulder arthroplasty components and surgical techniques that restore glenohumeral motion. J Should Elbow Surg 22(2):179-187

46. Walch G, Badet R, Boulahia A, Khoury A (1999) Morphologic study of the Glenoid in primary glenohumeral osteoarthritis. J Arthroplasty 14(6):756-760. doi:10.1016/S0883-5403(99)90232-2

47. Wall B, Nove-Josserand L, O'Connor DP, Edwards TB, Walch G (2007) Reverse total shoulder arthroplasty: a review of results according to etiology. J Bone Joint Surg Am 89(7):1476-1485

48. Werner CM, Steinmann PA, Gilbart M, Gerber C (2005) Treatment of painful pseudoparesis due to irreparable rotator cuff dysfunction with the Delta III reverse ball-and-socket total shoulder prosthesis. J Bone J Surg Am 87(7):1476-1486

49. De Wilde LF, Audenaert EA, Berghs BM (2004) Shoulder prostheses treating cuff tear arthropathy: a comparative biomechanical study. J Orthop Res 22(6):1222-1230

50. Young SM, Everts NM, Ball CM, Astley TM, Poon PC (2009) The SMR reverse shoulder prosthesis in the treatment of cuff-deficient sholuder conditions. J Should Elbow Surg 18(4):622-626. doi:10. 1016/j.jse.2009.01.017 